\section{(1) \\ CrossMark}

\title{
Precision medicine in acute respiratory distress syndrome: workshop report and recommendations for future research
}

\author{
Lieuwe D.J. Bos (1) ${ }^{1,2,3}$, Antonio Artigas ${ }^{4}$, Jean-Michel Constantin ${ }^{5}$, \\ Laura A. Hagens (10), Nanon Heijnen ${ }^{6}$, John G. Laffey (10,8, Nuala Meyer ${ }^{9}$, \\ Laurent Papazian $^{10}$, Lara Pisani ${ }^{11}$, Marcus J. Schultz (1) ${ }^{1,2,3}$, Manu Shankar-Hari (1) ${ }^{12}$, \\ Marry R. Smit ${ }^{1}$, Charlotte Summers (1) ${ }^{13}$, Lorraine B. Ware ${ }^{14}$, Raffaele Scala ${ }^{15}$ \\ and Carolyn S. Calfee ${ }^{16,17}$
}

@ERSpublications

Acute respiratory distress syndrome (ARDS) shows variation on three distinct levels: aetiological, physiological and biological. The lack of a common presentation implies that intervention studies in patients with ARDS need to be phenotype aware and apply a precision medicine approach. https://bit. ly/36XZWcP

Cite this article as: Bos LDJ, Artigas A, Constantin J-M, et al. Precision medicine in acute respiratory distress syndrome: workshop report and recommendations for future research. Eur Respir Rev 2021; 30: 200317 [https://doi.org/10.1183/16000617.0317-2020].

ABSTRACT Acute respiratory distress syndrome (ARDS) is a devastating critical illness that can be triggered by a wide range of insults and remains associated with a high mortality of around $40 \%$. The search for targeted treatment for ARDS has been disappointing, possibly due to the enormous heterogeneity within the syndrome. In this perspective from the European Respiratory Society research seminar on "Precision medicine in ARDS", we will summarise the current evidence for heterogeneity, explore the evidence in favour of precision medicine and provide a roadmap for further research in ARDS. There is evident variation in the presentation of ARDS on three distinct levels: 1) aetiological; 2) physiological and 3) biological, which leads us to the conclusion that there is no typical ARDS. The lack of a common presentation implies that intervention studies in patients with ARDS need to be phenotype aware and apply a precision medicine approach in order to avoid the lack of success in therapeutic trials that we faced in recent decades. Deeper phenotyping and integrative analysis of the sources of variation might result in identification of additional treatable traits that represent specific pathobiological mechanisms, or so-called endotypes.

\section{Introduction}

Acute respiratory distress syndrome (ARDS) is a devastating critical illness that can be triggered by a wide range of insults. It is characterised by leukocyte infiltration, local immune activation and alveolar endothelial and epithelial injury associated with increased pulmonary vascular permeability, acute pulmonary oedema, and loss of aerated lung tissue [1]. The diagnosis of ARDS is based on the development of bilateral opacities on chest radiography indicative of pulmonary oedema within 1 week of known clinical insult, in combination with impaired oxygenation as measured by the ratio of arterial to inspired oxygen $\left(P_{\mathrm{aO}_{2}} / F_{\mathrm{IO}_{2}}\right)$ despite the application of at least $5 \mathrm{~cm} \mathrm{H}_{2} \mathrm{O}$ of positive end-expiratory pressure (PEEP) [2]. Cardiac failure does not fully explain the radiographic and clinical abnormalities.

No specific aetiological, physiological or biological criteria are required for ARDS diagnosis, inherently resulting in heterogeneity on these three levels. The in-hospital mortality of ARDS remains around 30$40 \%$ [3]. Treatment of ARDS is limited to the prevention of harm induced by ventilatory support 
(ventilator-induced lung injury (VILI)) through lung protective mechanical ventilation strategies [4]. Recent interventional studies targeting the pathophysiological principles underlying ARDS, such as inflammation, coagulation, oxidative stress and endothelial injury, have failed to show benefit and, therefore, the therapy for ARDS remains supportive [5]. We hypothesise that these treatments failed partly due to insufficient understanding of the heterogeneity of ARDS.

Precision medicine has been defined as "treatments targeted to the needs of individual patients on the basis of genetic, biomarker, phenotypic, or psychosocial characteristics (a subphenotype) that distinguish a given patient from other patients with similar clinical presentations (the phenotype)" [6]. Precision medicine aims to provide a treatment to those patients who will benefit and withhold that same treatment from those who will not benefit or will be harmed. When does precision medicine provide additional information on top of the Oslerian paradigm of syndromic pattern recognition? This added value is more likely when the clinical diagnostic criteria do not capture a single pathophysiological process because only some of the patients will benefit from a treatment targeting a specific pathophysiological process. Simultaneously, other patients may experience side-effects without the potential for benefit. The profit from a precision medicine approach will increase when those side-effects have larger consequences, as is likely the case in critically ill patients. We acknowledge that "all models are wrong" and that any subphenotype can be further split into smaller units [7]. Therefore, we do not seek excessive elaboration to explain the pathophysiology of ARDS but rather seek a simple description of subphenotypes that are evaluated on their predictive accuracy for treatment response in the hope to find "models that are useful" and applicable in clinical practice [7].

In this perspective from the European Respiratory Society research seminar on "Precision medicine in ARDS", we will summarise the current evidence for heterogeneity, explore the evidence in favour of precision medicine and provide a roadmap for further research in ARDS. The basic assumption of the seminar was that there are challenges in heterogeneity of ARDS on three distinct levels: 1) aetiological, 2) physiological and 3) biological [8]. The experts attending the research seminars provided literature reviews, and researchers presented their latest paper in the form of abstracts. Focus groups worked on statements for each of the three domains, which were discussed in a round table format at the conference.

\section{Aetiology}

When ARDS was described in 1967 by AsHBAUgh et al. [9], it was noted that patients with different risk factors showed similar clinical characteristics, and post mortem pathological evaluation revealed diffuse alveolar damage (DAD) in all. ARDS is now known to be a frequent cause of acute respiratory failure, and subsequent definitions have become more and more inclusive, lumping patients with increasingly heterogeneous conditions together $[2,9,10]$. With the current definition, only a minority of patients who fulfil the criteria for ARDS actually have findings of DAD at post mortem pathological evaluation [11]. Aetiological factors may play role in the heterogeneity of ARDS at three levels: risk factors for lung injury, factors that further induce pulmonary oedema, and clinical states that stand in the way of reparative processes of lung injury [1].

Affiliations: ${ }^{1}$ Intensive Care, Amsterdam UMC - location AMC, University of Amsterdam, Amsterdam, The Netherlands. ${ }^{2}$ Laboratory of Intensive Care and Anesthesiology Amsterdam UMC - location AMC, University of Amsterdam, Amsterdam, The Netherlands. ${ }^{3}$ Dept of Respiratory Medicine, Amsterdam UMC - location AMC, University of Amsterdam, Amsterdam, The Netherlands. ${ }^{4}$ Critical Care Center, Corporació Sanitaria Universitaria Parc Tauli, CIBER Enfermedades Respiratorias, Autonomouus University of Barcelona, Sabadell, Spain. ${ }^{5}$ Dept of Anaesthesiology and Critical Care, Sorbonne University, GRC 29, AP-HP, DMU DREAM, PitiéSalpêtrière Hospital, Paris, France. ${ }^{6}$ Intensive care, Maastricht UMC, University of Maastricht, Maastricht, The Netherlands. ${ }^{7}$ Anaesthesia and Intensive Care Medicine, School of Medicine, and Regenerative Medicine Institute (REMEDI) at CÚRAM Centre for Research in Medical Devices, National University of Ireland Galway, Galway, Ireland. ${ }^{8}$ Dept of Anaesthesia, University Hospital Galway, Saolta Hospital Group, Galway, Ireland. ${ }^{9}$ Division of Pulmonary, Allergy, and Critical Care Medicine, University of Pennsylvania Perelman School of Medicine, Philadelphia, PA, USA. ${ }^{10}$ Intensive Care Medicine and regional ECMO center, North hospital - AixMarseille University, Marseille, France. ${ }^{11}$ Dipartimento Cardio-Toraco-Vascolare, Policlinico S.OrsolaMalpighi, Bologna, Italy. ${ }^{12}$ School of Immunology \& Microbial Sciences, Kings College London, London, UK.

${ }^{13}$ Dept of Medicine, University of Cambridge, Cambridge, UK. ${ }^{14}$ Vanderbilt University Medical Center, Nashville, TN, USA. ${ }^{15}$ Respiratory Division with Pulmonary Intensive Care Unit, S. Donato Hospital, Usl Toscana Sudest, Arezzo, Italy. ${ }^{16}$ Division of Pulmonary, Critical Care, Allergy and Sleep Medicine, Dept of Medicine, University of California, San Francisco, CA, USA. ${ }^{17}$ Dept of Anesthesia, University of California, San Francisco, CA, USA.

Correspondence: Lieuwe D.J. Bos, Intensive Care, Academic Medical Center - University of Amsterdam, Meibergdreef 9, M0-127, Amsterdam 1105AZ, The Netherlands. E-mail: l.d.bosdamc.uva.nl 
Evidence for heterogeneity and precision approaches

Treatment targeted at specific aetiological causes for ARDS

Other aetiologies under the umbrella of "diffuse interstitial acute lung diseases", "diffuse pulmonary infections" and "drug/chemical-induced diffuse lung disease" can present with similar characteristics as ARDS and fall within the syndrome definition. These diagnoses are sometimes referred to as ARDS-mimics because they show a known and distinct pathophysiology and require specific treatment. These patients may be included in clinical trials if no additional diagnostic tests are performed to distinguish between ARDS and ARDS-mimics [12]. This issue may be important for clinical practice and trial design, as some of these ARDS-mimics can be effectively treated with immunosuppressants such as corticosteroids, antimicrobial drugs or withholding the drug that caused the lung injury. There is no consistent diagnostic approach that takes the probabilities of ARDS-mimics into account [13].

The specific causes for infectious and drug/chemical-induced diffuse lung disease may differ between institutions and can change over time, as is exemplified by the recent rise of vaping-induced lung injury [14] and the SARS-CoV-2-2019 pandemic [15]. Recognition of the underlying cause is essential because one of the fundamentals of ARDS care is the adequate treatment of the underlying disease, when possible. The rapid discovery of dexamethasone as effective treatment for severe COVID-19 pneumonia shows the importance of recognition of the underlying aetiology and the potential for phenotype-aware clinical trials [16].

\section{Aggravation of lung injury}

We also recognise that there are patients with risk factors for ARDS in whom pulmonary oedema is caused or aggravated via the administration of fluids, transfusions or injurious mechanical ventilation [17-20]. This iatrogenic injury should be considered a second hit after the first insult and adds additional complexity to the syndrome.

ARDS rapidly resolves in some patients, while it does not in others [1, 21, 22]. Super-imposed nosocomial infection and inadequate or suboptimal treatment of the primary insult may contribute to the persistence of lung injury. In some patients, fibroproliferation might contribute to the unfavourable evolution of ARDS, and there currently is no proven therapy to counteract this process. Some evidence suggests that corticosteroid therapy might be beneficial specifically in patients with fibroproliferation [23].

\section{Attributable mortality}

The attributable mortality of ARDS on top of chronic comorbidities and the underlying aetiological factors is unknown [24]. The attributable mortality may be different between patients with different risk factors. Trauma and sepsis patients with ARDS required more resources and died more frequently than patients with similar injuries but no ARDS $[25,26]$. However, in patients with severe community-acquired pneumonia, ARDS was not a risk factor for mortality [27].

\section{Research agenda}

1) Establish and apply a diagnostic protocol to identify treatable diseases within the syndrome diagnosis of ARDS.

2) Evaluate the contribution of second hits such as mechanical ventilation, excess fluid administration and blood product transfusions, to specific aetiological phenotypes.

3) In patients with unresolved ARDS, identify those patients who have fibroproliferation/nosocomial infection, or persistence of the primary cause of ARDS.

4) In patients with evidence for fibroproliferation, test treatments such as steroids or anti-fibrotic treatments in clinical trials.

5) Quantify the attributable mortality of ARDS in the setting of different aetiologies.

6) When performing randomised controlled trials (RCTs), particular care needs to be taken to identify and, depending on the intervention of choice, possibly exclude patients with ARDS-mimic diagnoses.

\section{Physiology and morphology}

The aim of mechanical ventilation in ARDS is to improve gas exchange and thereby buy time for treatment of the underlying cause, resolution of pulmonary oedema, and repair of injured lung epithelium and endothelium [28]. The main challenge is to prevent further damage to the lungs due to VILI. Low tidal volume ventilation was shown to be protective in all patients with ARDS, sparking interest in the application of other strategies that were considered "lung protective" in an unselected cohort of patients such as recruitment manoeuvres, high PEEP and prone positioning [29]. "Protective" ventilator strategies other than low tidal volume ventilation are likely to work in some subsets of patients, while they could be ineffective or even harmful in others [30, 31]. 
Evidence for heterogeneity and precision approaches

Noninvasive support of ventilation and oxygenation

Around $16 \%$ of patients with ARDS may not require invasive mechanical ventilation but could be managed by noninvasive mechanical ventilation (NIV) or high-flow nasal oxygen (HFNO) therapy [32]. High tidal volumes and strongly negative intrathoracic pressures during NIV are speculated to contribute to failure of this approach via aggravation of lung injury through large pulmonary pressure swings, sometimes referred to as patient self-inflicted lung injury (P-SILI) $[33,34]$ and might be more frequent in patients with moderate or severe hypoxaemia $\left(P_{\mathrm{aO}_{2}} / F_{\mathrm{IO}_{2}}<200 \mathrm{mmHg}\right)$. The helmet interface is suggested to be most effective in patients with acute respiratory failure, many of whom have ARDS [35]. HFNO might, however, be more beneficial in patients with moderate or severe hypoxaemia [36]. Patients managed with NIV or HFNO whose clinical conditions do not stabilise with this treatment and who require intubation have worse outcomes [37]. Since the COVID-19 pandemic, there is increasing interest in combining HFNO or helmet NIV with prone positioning [38].

\section{Persistence of hypoxaemia}

The degree of hypoxaemia indicated by the $P_{\mathrm{aO}_{2}} / F_{\mathrm{IO}_{2}}$ and PEEP at the moment of diagnosis may be less predictive for mortality than a reclassification on the following day [39-41]. There seems to be a phenotype of ARDS that resolves rapidly [42]. It is unclear what underlying physiology explains this phenotype, and mortality in this population is still high [39].

\section{Recruitment response}

There is a preferential treatment response to higher PEEP, prone positioning and early neuromuscular blockade in patients with lower $P_{\mathrm{aO}} / F_{\mathrm{IO}_{2}}$ ratios [43-46]. However, despite these data, enrichment based on severe hypoxaemia has proven insufficiently effective for an "open lung approach" with high PEEP and aggressive recruitment manoeuvres, and for neuromuscular blockade in recent years $[47,48]$.

There is considerable variation in the amount of recruitable lung tissue between patients with ARDS. Predictors for a recruitable lung include: nonpulmonary ARDS, nonfocal ARDS [51] and lower $P_{\mathrm{aO}_{2}} / F_{\mathrm{IO}_{2}}$ ratios [52]. Thus, there is heterogeneity in response to an open lung approach between patients with different phenotypes. A recent RCT assigned patients to a "standard" lung protective mechanical ventilation protocol or to a personalised approach based on a focal or nonfocal morphology of consolidations on computed tomography (CT)-imaging of the lung [53]. The intention to treat analysis did not show a difference between the arms, possibly because of the misclassification of morphological patterns in $20 \%$ of cases. In the per protocol analysis excluding misclassified patients, patients in the personalised mechanical ventilation protocol group showed a lower mortality (hazard ratio 0.6, 95\% CI 0.36-0.99). Interestingly, patients who were misclassified had a significantly higher mortality when receiving the inappropriate treatment, suggesting harm of these interventions when applied to the wrong population and highlighting the importance of precision therapy.

Studies have failed to address the interactions between tidal volume, PEEP, driving pressure and respiratory rate and, rather, evaluated each variable in isolation. The establishment of the concept of mechanical power may serve as a way to stratify the amount of damage inflicted by mechanical ventilation $[49,50]$.

\section{Research agenda}

1) Identify phenotypes that are predictive for a positive response to HFNO or NIV.

2) Identify patients who benefit from specific NIV settings (flow, inspiratory pressure and PEEP), interface, sedation and analgesia to limit p-SILI.

3) Improve reclassification of patients after the first period of treatment to better identify rapid resolvers who may not need further lung-focused therapy.

4) Develop alternative (imaging) modalities that can accurately identify focal and nonfocal ARDS morphology.

5) When performing RCTs, collect data on the physiological and morphological phenotype of patients in the study and evaluate differential treatment effects.

\section{Biology}

Lung injury in ARDS is a consequence of a complex interaction between cellular and biochemical pathways resulting in lung epithelial and endothelial damage that is clinically characterised by protein-rich pulmonary oedema [54]. Many underlying biochemical processes have been targeted with drugs that effectively reverse the molecular mechanism of interest, but none of the studied drugs were effective in lowering mortality [5]. Heterogeneity in the biological mechanisms responsible for lung injury may explain these results. Even if patients with ARDS on average have an increased concentration of a certain cell or 
molecule, indicative for a specific mechanistic contribution to the development of lung injury, it does not necessarily mean that every ARDS patient would benefit from targeting the underlying pathway. In fact, targeting a pathway that is not activated in a specific patient might lead to harm. Therefore, a personal assessment of systemic and pulmonary host response could help identifying homogenous patient groups and guide treatment decisions.

\section{Evidence for heterogeneity and precision approaches}

Pre-clinical research

In an ideal world, an experimental model of ARDS would fully reflect the mechanisms of injury, the clinical evolution, and the outcomes; in this sense, no experimental model of acute lung injury comes close [55]. No experimental model can hope to replicate the complexity of human ARDS, but some models will reflect well some aspect(s) of the syndrome [56]. More recently, comparison of biological heterogeneity between septic humans and mice has resulted in the validation of subphenotypes across species [57].
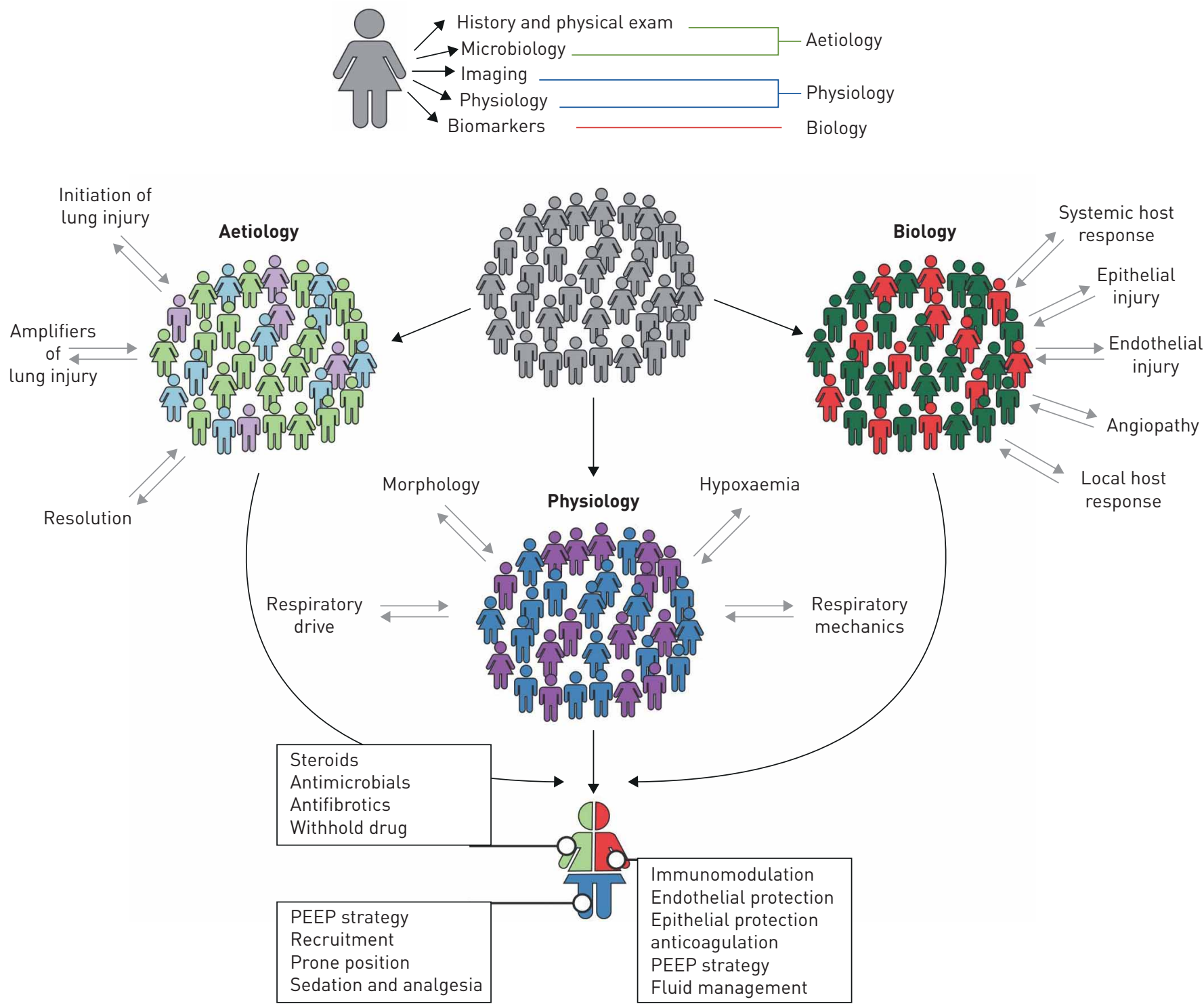

FIGURE 1 ARDS can be phenotyped based on information in three domains: aetiology, physiology and biology. Multiple phenotypes can coexist between and within a domain (within domain coexistence not shown). We recognise that there is a complex interaction between phenotypes and that true endotypes might capture phenotypic presentations within multiple domains. Traits are potentially treatable based on phenotypic recognition and subsequent empirical evidence for effectivity of intervention or on deep understanding of the critical causal pathways. ARDS: acute respiratory distress syndrome; PEEP: positive end-expiratory pressure. 
TABLE 1 Potential treatable traits in ARDS across aetiology, physiology and morphology, and biology

\begin{tabular}{|c|c|c|c|c|c|c|}
\hline Domain & Subdomain & Trait & Test & Evidence & Interventions to be tested & Challenges \\
\hline \multirow[t]{18}{*}{ Aetiology } & Causal pathogen & COVID-19 & PCR for virus & [16] & Dexamethasone & \\
\hline & ARDS-mimic & $\begin{array}{l}\text { Diffuse acute interstitial lung } \\
\text { diseases }\end{array}$ & $\begin{array}{l}\text { History } \\
\text { Imaging }\end{array}$ & [75-77] & Immunosuppression & $\begin{array}{l}\text { Relatively rare and requires systematic investigation to } \\
\text { identify }\end{array}$ \\
\hline & & Diffuse nulmonary infections & $\begin{array}{l}\text { Immunological analysis } \\
\text { History }\end{array}$ & & & \\
\hline & & Diffuse pulmonary infections & History & [78] & Antimicrobials & \\
\hline & & & $\begin{array}{l}\text { Serology } \\
\text { Imaging }\end{array}$ & & & \\
\hline & & & Culture & & & \\
\hline & & & Metabolic products & & & \\
\hline & & & Metagenomics & & & \\
\hline & & Drug-induced diffuse lung disease & History & www.pneumotox.com & Withhold drug & \\
\hline & Amplifiers of lung & Fluid overload & History & [79] & Diuretics & Diagnosis of fluid overload can be challenging \\
\hline & injury & & $\begin{array}{l}\text { Clinical examination } \\
\text { Ultrasound }\end{array}$ & & Vasopressors & \\
\hline & & & Extravascular lung water & & & \\
\hline & & Ventilator-induced lung injury & Tidal volume & [80] & Lower tidal volumes & No direct test for the actual development of VILI \\
\hline & & & Driving pressure & & & \\
\hline & & & Mechanical power & & & \\
\hline & $\begin{array}{l}\text { Nonresolving lung } \\
\text { injury }\end{array}$ & Fibroproliferation & $\begin{array}{l}\text { Markers of fibroproliferation in } \\
\text { bronchoalveolar lavage fluid }\end{array}$ & [27] & $\begin{array}{l}\text { Corticosteroids } \\
\text { Antifibrotics }\end{array}$ & Biomarker test not routinely available \\
\hline & & Secondary infection & $\begin{array}{l}\text { Imaging } \\
\text { Culture }\end{array}$ & [81] & Antimicrobials & $\begin{array}{l}\text { Identify ventilator-associated pneumonia in patient } \\
\text { with ARDS }\end{array}$ \\
\hline & & & Metagenomics & & & \\
\hline \multirow[t]{6}{*}{ Physiology } & Shunt & $P_{\mathrm{aO}_{2}} / F_{\mathrm{IO}_{2}}$ & Blood gas & {$[43,44]$} & Prone positioning & Various thresholds proposed in different studies \\
\hline & & & & & $\begin{array}{l}\text { Adjust PEEP } \\
\text { Lung recruitment }\end{array}$ & Influence of PEEP on $P_{\mathrm{aO}_{2}} / F_{\mathrm{IO}_{2}}$ \\
\hline & Ventilation & Dead space ventilation & $\begin{array}{l}\text { Dead space calculation } \\
\text { Ventilatory ratio }\end{array}$ & [82] & Adjust PEEP & Volumetric capnography not widely available \\
\hline & Drive & High respiratory drive on NIV & Oesophageal pressure & [34] & Analgesia and sedation & Balance between high drive and too low drive \\
\hline & Mechanics & High mechanical power & Formula based & [49] & $\begin{array}{l}\text { Adjust PEEP, tidal volume and/or } \\
\text { respiratory rate }\end{array}$ & $\begin{array}{l}\text { Various thresholds proposed and unclear how to } \\
\text { adjust settings based on value }\end{array}$ \\
\hline & & Driving pressure & $\begin{array}{l}\text { Ventilator settings } \\
\text { Oesophageal pressure }\end{array}$ & [83] & Adjust PEEP & Various thresholds proposed in different studies \\
\hline \multirow[t]{2}{*}{ Morphology } & Imaging & Focal & Imaging & {$[53,84,85]$} & $\begin{array}{l}\text { Prone positioning } \\
\text { Low PEEP }\end{array}$ & $\begin{array}{l}\text { Misclassification of morphology common and } \\
\text { associated with worse outcome }\end{array}$ \\
\hline & & Nonfocal & Imaging & {$[53,84,85]$} & $\begin{array}{l}\text { Lung recruitment } \\
\text { High PEEP }\end{array}$ & \\
\hline \multirow{6}{*}{ Biology } & Systemic host & Hyperinflammatory (or Reactive) & IL-8, bicarbonate and protein C & {$[31,60,62,63,86]$} & High PEEP & No routine test available \\
\hline & response & & IL-6, bicarbonate and TNFRI & & $\begin{array}{l}\text { Restrictive fluid } \\
\text { Simvastatin } \\
\text { Immunomodulation }\end{array}$ & Frequently unknown if cause or effect of lung injury \\
\hline & Epithelial injury & Damaged epithelium & Biomarkers e.g. sRAGE & [87] & Epithelial protection & \\
\hline & Endothelial injury & $\begin{array}{l}\text { Vascular permeability and } \\
\text { endothelial injury }\end{array}$ & Biomarkers e.g. angiopoietin 1 and 2 & [88] & $\begin{array}{l}\text { Endothelial protection } \\
\text { Immunomodulation }\end{array}$ & \\
\hline & Angiopathy & Microthrombosis & $\begin{array}{l}\text { Biomarkers e.g. D-dimers, PAl-1 } \\
\text { Perfusion imaging }\end{array}$ & {$[89,90]$} & $\begin{array}{l}\text { Anticoagulation } \\
\text { Immunomodulation }\end{array}$ & \\
\hline & Local host response & Pulmonary hyper-inflammation & $\begin{array}{l}\text { Biomarkers in bronchoalveolar lavage } \\
\text { fluid }\end{array}$ & [91] & Immunomodulation & \\
\hline
\end{tabular}

There are a wide range of clinical conditions, ARDS severities and mediators in lung injury pathogenesis that may be targetable for treatment. Most interventions listed are speculative and should not yet be applied. The list is also not exhaustive. For all these interventions, we emphasise the need for phenotype-aware randomised controlled trials. COVID-19: coronavirus disease 2019; ARDS: acute respiratory distress syndrome; VILI: ventilator-induced lung injury; $P_{a O_{2}}$ : arterial oxygen tension; FIO ${ }_{2}$ : inspiratory oxygen fraction; PEEP: positive end-expiratory pressure; NIV: noninvasive mechanical ventilation; IL: interleukin; TNFRI: tumour necrosis factor receptor 1; sRAGE: soluble receptor for advanced glycation endproducts; PAl-1: plasminogen activator inhibitor-1. 
Identification of biological phenotypes

Using latent class analysis (LCA) of a panel of plasma biomarkers of inflammation, endothelial injury and coagulopathy in combination with routinely available clinical variables revealed two phenotypes across five RCTs [31, 58-60]. The so-called hyperinflammatory phenotype consistently had a higher mortality than the hypoinflammatory phenotype. These phenotypes seem to be stable at least up to 3 days after intensive care unit (ICU) admission [61].

Cluster analysis of a set of 20 biomarkers of inflammation, endothelial injury and coagulopathy without consideration of routinely available clinical variables in an observational study also revealed two phenotypes with consistent differences in mortality [62]. The gene expression of leukocytes in peripheral blood at admission to the ICU was profoundly different between phenotypes particularly in pathways involved in neutrophil activation, cholesterol metabolism and oxidative phosphorylation [63].

\section{Differential treatment responses between biological phenotypes}

Post hoc analysis of several RCTs showed that patients with the hyperinflammatory phenotype had a differential response to PEEP, fluid management and simvastatin treatment compared with patients with a hypoinflammatory phenotype [31, 58-60].

A parsimonious model of different combinations of three or four biomarkers (interleukin (IL)-6 or IL-8, bicarbonate, tumour necrosis factor receptor 1 or protein $\mathrm{C}$, possibly in combination with vasopressor use) as well as a machine learning algorithm that used readily available clinical data was able to accurately identify the LCA phenotypes and showed adequate predictive enrichment in post hoc analysis of the above described RCTs $[64,65]$.

\section{Endotyping}

Despite these promising findings, the currently available data is insufficient to endotype because subphenotypes have not been related to specific pathobiological mechanisms $[66,67]$. True endotyping would reliably identify and measure the critical biochemical pathways within patients and inform treatment based on a deep and highly predictive understanding of the underlying pathophysiology.

\section{Research agenda}

1) Translate biological variation in humans to animal models of acute lung injury by matching animal models to phenotypes rather than assuming that the model is representative of all human ARDS.

2) Embrace heterogeneity within animal models by introducing variation through experimentation in different laboratories, across (sub-)species, ages and comorbidities.

3) Validate the identified biological phenotypes in prospective clinical studies.

4) Determine the evolution of biological phenotypes throughout the course of ARDS and evaluate the influence of interventions on phenotype assignment.

5) Explore the generalizability of biological phenotypes of ARDS to other populations such as patients with acute respiratory failure, sepsis and unselected ICU patients.

6) Compare phenotypes based on plasma biomarkers to the pulmonary biological response by obtaining simultaneous samples from the lung and systemic compartments.

7) Further increase our understanding of heterogeneity within ARDS by deeper phenotyping and better understanding the immunology through functional assays, possibly resulting in the identification of endotypes.

8) Develop surrogate outcomes that reflect effectiveness of treatment within the target pathway of intervention.

9) Ideally, all ARDS RCTs should collect biological samples in order to consider the biological phenotype of patients in the study and evaluate differential treatment effects.

\section{Integrating heterogeneity in aetiology, physiology and biology}

One of the major challenges of precision medicine is to split patients into homogenous groups that are adequately enriched for positive treatment effects, while lumping a sufficient proportion of patients together to test interventions on clinically relevant outcomes, which frequently require large sample sizes. For this purpose, it is pivotal to understand the links between the three sources of heterogeneity (figure 1). We recognise that any subdivision in domains, including ours in aetiology, physiology and biology, is arbitrary. If a phenotype translates from one domain to another, the more easily evaluated phenotype can be used as a surrogate for the other. For example, the plasma concentration of the soluble form of the receptor for advanced glycation end products (sRAGE), a marker for lung epithelial injury, is strongly associated with the presence of nonfocal ARDS [68]. This finding is in line with the hypothesis that widespread pulmonary permeability is more profound in nonfocal ARDS and that an open lung approach 
may benefit these patients. Furthermore, sRAGE or another surrogate may substitute for CT scanning to identify this morphological phenotype. However, biomarkers of endothelial injury and lung epithelial damage were significantly different between patients with pulmonary and nonpulmonary causes of lung injury, but still showed so much overlap that we cannot equate the aetiological factor with a particular biological phenotype $[69,70]$. We recognise these are only the first steps into integrating information from the different domains and acknowledge the need for more collaboration between experts in all aspects of the quantification of heterogeneity and integration of the generated information in the near future.

\section{Research agenda}

1) Define the relationship between aetiological, physiological and biological phenotypes, with deeper phenotyping on all three levels.

2) A better treatment of ARDS requires open sharing of data, expertise and integration of multiple data streams.

3) Apply techniques used to integrate phenotypic data in other complex syndromes, such as chronic airway diseases or heart failure, in ARDS [71].

\section{Towards treatable traits}

One approach that has gained considerable attention in chronic airway diseases is the concept of treatable traits [72]. To cite: "these traits can be treatable based on phenotypic recognition (and thereby probabilistic evidence based on positive and negative predictive values) or on deep understanding of the critical causal pathways (e.g. true endotypes)" [72]. These treatable traits may be independent of the traditional syndromic diagnosis and may change over time. For ARDS, we propose to take a similar approach in which a patient can fulfil multiple treatable traits and thereby require multiple treatments. Furthermore, as treatable traits are, per definition, label-free they might generalise outside of ARDS, to acute respiratory failure [73], sepsis [74] or even unselected critically ill ICU patients. We can already start to recognise some potential treatable traits within the ARDS syndrome (table 1), although we emphasise that prospective validation of these approaches is pivotal. Using a treatable traits framework, we can rethink the concept of "ARDS-mimickers" and consider them as examples of treatable traits within the larger syndrome of ARDS (see table 1).

\section{Implications of the research agenda}

The evidence for aetiological, physiological and biological heterogeneity in ARDS has important implications for clinical, translational and basic scientists. We presented a list of research priorities that will require elaborate collaboration between researchers across continents, disciplines and expertise. We invite researchers and clinicians from around the world to join us in these efforts. One of the major challenges is that funding agencies incentivise solo projects in radical new directions rather than the highly collaborative, descriptive and iterative studies that are needed to better define phenotypes and validate previous findings [92]. This paper will require novel initiatives of international and intercontinental collaboration.

Perhaps the most important implication of the observed heterogeneity in ARDS is that future RCTs in an unselected population of patients with ARDS should make efforts to thoroughly phenotype the included patients, so as to try to identify phenotypes that are predictive of a positive response in post hoc analysis. Ultimately, we believe that RCTs should include a phenotype-based allocation strategy in order to have a chance of showing benefit of the intervention, though it is premature at this point to proceed in this direction without prospective validation of phenotypes. Furthermore, it is of utmost importance to select the end-point based on the attributable effect of ARDS on the outcome within the specific phenotype.

\section{Conclusions}

The search for targeted treatment for ARDS has been disappointing, possibly due to the enormous heterogeneity within the syndrome. We propose to systematically address the variations in aetiology, physiology and biology in order to identify treatable traits that can be targeted in future clinical trials. We have established a research agenda and a list of potential treatable traits that may serve as a basis for future studies. Deeper phenotyping and integrative analysis of the sources of variation might result in identification of additional treatable traits that represent specific pathobiological mechanisms, or so-called endotypes.

Conflict of interest: L.D.J. Bos reports grants from the Dutch Lung Foundation (Young investigator grant), the Dutch Lung Foundation and Health Holland (Public-Private Partnership grant) and the Dutch Lung Foundation (Dirkje Postma Award), and grants from IMI COVID19 imitative and Amsterdam UMC Fellowship, outside the submitted work. A. Artigas reports grants from Grifols and Fisher \& Paykel, and personal fees from Grifols, outside the submitted 
work. J-M. Constantin has nothing to disclose. L.A. Hagens has nothing to disclose. N. Heijnen has nothing to disclose. J.G. Laffey reports grants from Science Foundation Ireland and Health Research Board Ireland, outside the submitted work. N. Meyer reports grants from National Institutes of Health, outside the submitted work. L. Papazian has nothing to disclose. L. Pisani reports personal fees from Fisher \& Paykel, outside the submitted work. M.J. Schultz has nothing to disclose. M. Shankar-Hari has nothing to disclose. M.R. Smit has nothing to disclose. C. Summers reports grants from GlaxoSmithKline and AstraZeneca, outside the submitted work. L.B. Ware reports grants from National Institutes of Health, during the conduct of the study; other funding from CSL Behring, Genentech and Global Blood Therapeutics, and personal fees from Merck, Bayer and Quark, outside the submitted work. R. Scala has nothing to disclose. C. Calfee reports grants from NIH, during the conduct of the study; grants and personal fees from Roche/ Genentech and Bayer, personal fees from Quark Pharmaceuticals, Prometic, Genle Life Sciences and Vasomune, outside the submitted work.

Support statement: Funding has been received from the Amsterdam UMC Fellowship. L.D.J. Bos is supported by the Dutch Lung Foundation (Longfonds) through the Young Investigator Award, the Dirkje Postma Award and a publicprivate partnership grant. M. Shankar-Hari is supported by the National Institute for Health Research Clinician Scientist Award (CS-2016-16-011). The views expressed in this publication are those of the author(s) and not necessarily those of the NHS, the National Institute for Health Research or the Dept of Health and Social Care. C. Calfee is supported, in part, by NIH HL140026. Funding information for this article has been deposited with the Crossref Funder Registry.

\section{References}

1 Thompson B, Chambers R, Liu K. Acute respiratory distress syndrome. N Engl J Med 2017; 377: 562-572.

2 ARDS Definition Task Force, Ranieri VM, Rubenfeld GD, et al. Acute respiratory distress syndrome: the Berlin definition. JAMA 2012; 307: 2526-2533.

3 Bellani G, Laffey JG, Pham T, et al. Epidemiology, patterns of care, and mortality for patients with acute respiratory distress syndrome in intensive care units in 50 countries. JAMA 2016; 315: 788-800.

4 Fan E, Brodie D, Slutsky AS. Acute respiratory distress syndrome. JAMA 2018; 319: 698-710.

5 Boyle AJ, McNamee JJ, McAuley DF. Biological therapies in the acute respiratory distress syndrome. Expert Opin Biol Ther 2014; 14: 969-981.

6 Jameson JL, Longo DL. Precision medicine-personalized, problematic, and promising. N Engl J Med 2015; 372: 2229-2234.

$7 \quad$ Box GEP. Science and statistics. J Am Stat Assoc 1976; 71: 791.

8 Sinha P, Calfee CS. Phenotypes in acute respiratory distress syndrome. Curr Opin Crit Care 2019; 25: 12-20.

9 Ashbaugh DG, Bigelow DB, Petty TL, et al. Acute respiratory distress in adults. Lancet 1967; 2: 319-323.

10 Bernard GR, Artigas A, Brigham KL, et al. The American-European Consensus Conference on ARDS. Definitions, mechanisms, relevant outcomes, and clinical trial coordination. Am J Respir Crit Care Med 1994; 20: 818-824.

11 Thille AW, Esteban A, Fernández-Segoviano P, et al. Comparison of the Berlin definition for acute respiratory distress syndrome with autopsy. Am J Respir Crit Care Med 2013; 187: 761-767.

12 Parrot A, Brun-Buisson C, Gibelin A, et al. Acute respiratory distress syndrome mimickers lacking common risk factors of the Berlin definition. Intensive Care Med 2016; 42: 164-172.

13 Papazian L, Calfee CS, Chiumello D, et al. Diagnostic workup for ARDS patients. Intensive Care Med 2016; 42: 674-685.

14 Layden JE, Ghinai I, Pray I, et al. Pulmonary illness related to E-cigarette use in Illinois and Wisconsin preliminary report. N Engl J Med 2020; 382: 903-916.

$15 \mathrm{Xu} \mathrm{Z}$, Shi L, Wang Y, et al. Case report: pathological findings of COVID-19 associated with acute respiratory distress syndrome. Lancet Respir 2020; 2600: 19-21.

16 RECOVERY Collaborative Group, Horby P, Lim WS, et al. Dexamethasone in hospitalized patients with Covid-19: preliminary report. N Engl J Med 2020; in press. [https://doi.org/10.1056/NEJMoa2021436]

17 Oakley C, Koh M, Baldi R, et al. Ventilation following established ARDS: a preclinical model framework to improve predictive power. Thorax 2019; 74: 1120-1129.

18 Dreyfuss D, Saumon G. Ventilator-induced lung injury: lessons from experimental studies. Am J Respir Crit Care Med 1998; 157: 294-323.

19 Toy P, Popovsky MA, Abraham E, et al. Transfusion-related acute lung injury: definition and review. Crit Care Med 2005; 33: 721-726.

20 Ahmed AH, Litell JM, Malinchoc $\mathrm{M}$, et al. The role of potentially preventable hospital exposures in the development of acute respiratory distress syndrome: a population-based study. Crit Care Med 2014; 42: 31-39.

21 Guerin C, Bayle F, Leray V, et al. Open lung biopsy in nonresolving ARDS frequently identifies diffuse alveolar damage regardless of the severity stage and may have implications for patient management. Intensive Care Med 2015; 41: 222-230

22 Gerard L, Bidoul T, Castanares-Zapatero D, et al. Open lung biopsy in nonresolving acute respiratory distress syndrome commonly identifies corticosteroid-sensitive pathologies, associated with better outcome. Crit Care Med 2018; 46: 907-914.

23 Steinberg KP, Hudson LD, Goodman RB, et al. Efficacy and safety of corticosteroids for persistent acute respiratory distress syndrome. N Engl J Med 2006; 354: 1045-1057.

24 Fuchs L, Feng M, Novack V, et al. The effect of ARDS on survival: do patients die from ARDS or with ARDS? J Intensive Care Med 2019; 34: 374-382.

25 Park PK, Cannon JW, Ye W, et al. Incidence, risk factors, and mortality associated with acute respiratory distress syndrome in combat casualty care. J Trauma Acute Care Surg 2016; 81: S150-S156.

26 Auriemma CL, Zhuo H, Delucchi K, et al. Acute respiratory distress syndrome-attributable mortality in critically ill patients with sepsis. Intensive Care Med 2020; 46: 1222-1231.

27 Cilloniz C, Ferrer M, Liapikou A, et al. Acute respiratory distress syndrome in mechanically-ventilated patients with community-acquired pneumonia. Eur Respir J 2018; 51: 1702215. 

traditional tidal volumes for acute lung injury and the acute respiratory distress syndrome. N Engl J Med 2000; 342: 1301-1308

29 Fan E, Del Sorbo L, Goligher EC, et al. An official American Thoracic Society/European Society of Intensive Care Medicine/Society of Critical Care Medicine Clinical Practice guideline: mechanical ventilation in adult patients with acute respiratory distress syndrome. Am J Respir Crit Care Med 2017; 195: 1253-1263.

30 Jabaudon M, Blondonnet R, Audard J, et al. Recent directions in personalised acute respiratory distress syndrome medicine. Anaesth Crit Care Pain Med 2018; 37: 251-258.

31 Calfee CS, Delucchi K, Parsons PE, et al. Subphenotypes in acute respiratory distress syndrome: latent class analysis of data from two randomised controlled trials. Lancet Respir Med 2014; 2: 611-620.

32 Bellani G, Laffey JG, Pham T, et al. Non-invasive ventilation of patients with ARDS: insights from the LUNG SAFE study. Am J Respir Crit Care Med 2017; 195: 67-77.

33 Brochard L. Ventilation-induced lung injury exists in spontaneously breathing patients with acute respiratory failure: yes. Intensive Care Med 2017; 43: 250-252.

34 Brochard L, Slutsky A, Pesenti A. Mechanical ventilation to minimize progression of lung injury in acute respiratory failure. Am J Respir Crit Care Med 2017; 195: 438-442.

35 Ferreyro BL, Angriman F, Munshi L, et al. Association of noninvasive oxygenation strategies with all-cause mortality in adults with acute hypoxemic respiratory failure. JAMA 2020; 324: 57-67.

36 Frat J-P, Thille AW, Mercat A, et al. High-flow oxygen through nasal cannula in acute hypoxemic respiratory failure. N Engl J Med 2015; 372: 2185-2196.

37 Laffey JG, Bellani G, Pham TT, et al. Potentially modifiable factors contributing to outcome from acute respiratory distress syndrome: the LUNG SAFE study. Intensive Care Med 2016; 42: 1865-1876.

38 Coppo A, Bellani G, Winterton D, et al. Feasibility and physiological effects of prone positioning in non-intubated patients with acute respiratory failure due to COVID-19 (PRON-COVID): a prospective cohort study. Lancet Respir 2020; 19: 1-10.

39 Madotto F, Pham T, Bellani G, et al. Resolved versus confirmed ARDS after 24 h: insights from the LUNG SAFE study. Intensive Care Med 2018; 44: 564-577.

40 Villar J, Fernández RL, Ambrós A, et al. A clinical classification of the acute respiratory distress syndrome for predicting outcome and guiding medical therapy. Crit Care Med 2015; 43: 346-353.

41 Bos LD, Cremer OL, Ong DSY, et al. External validation confirms the legitimacy of a new clinical classification of ARDS for predicting outcome. Intensive Care Med 2015; 41: 2004-2005.

42 Schenck EJ, Oromendia C, Torres LK, et al. Rapidly improving ARDS in therapeutic randomized controlled trials. Chest 2019; 155: 474-482.

43 Briel M, Meade M, Mercat A, et al. Higher vs lower positive end-expiratory pressure in patients with acute lung injury and acute respiratory distress syndrome: systematic review and meta-analysis. JAMA 2010; 303: 865-873.

44 Guérin C, Reignier J, Richard J-C, et al. Prone positioning in severe acute respiratory distress syndrome. $\mathrm{N}$ Engl $\mathrm{J}$ Med 2013; 368: 2159-2168.

45 Papazian L, Forel JM, Gacouin A, et al. Neuromuscular blockers in early acute respiratory distress syndrome. N Engl J Med 2010; 363: 1107-1116.

46 Guerin C. Prone ventilation in acute respiratory distress syndrome. Eur Respir Rev 2014; 23: 249-257.

47 The National Heart Lung institute and Blood Institute PETAL Clinical Trials Network. Early neuromuscular blockade in the acute respiratory distress syndrome. N Engl J Med 2019; 380: 1997-2008.

48 Cavalcanti AB, Suzumura ÉA, Laranjeira LN, et al. Effect of lung recruitment and titrated positive end-expiratory pressure (PEEP) vs low PEEP on mortality in patients with acute respiratory distress syndrome - a randomized clinical trial. JAMA 2017; 318: 1335-1345.

49 Gattinoni L, Tonetti T, Cressoni M, et al. Ventilator-related causes of lung injury: the mechanical power. Intensive Care Med 2016; 42: 1567-1575.

50 Vassalli F, Pasticci I, Romitti F, et al. Does iso-mechanical power lead to iso-lung damage? An experimental study in a porcine model. Anesthesiology 2020; 132: 1126-1137.

51 Pelosi P, D'Onofrio D, Chiumello D, et al. Pulmonary and extrapulmonary acute respiratory distress syndrome are different. Eur Respir J 2003; 22: Suppl. 42, 48s-56s.

52 Gattinoni L, Caironi P, Cressoni M, et al. Lung recruitment in patients with the acute respiratory distress syndrome. N Engl J Med 2006; 354: 1775-1786.

53 Constantin J-M, Jabaudon M, Lefrant J-Y, et al. Personalised mechanical ventilation tailored to lung morphology versus low positive end-expiratory pressure for patients with acute respiratory distress syndrome in France (the LIVE study): a multicentre, single-blind, randomised controlled trial. Lancet Respir Med 2019; 2600: 1-12.

54 Matthay MA, Zemans RL, Zimmerman GA, et al. Acute respiratory distress syndrome. Nat Rev Dis Primers 2019; 5: 18 .

55 Matute-Bello G, Frevert CW, Martin TR. Animal models of acute lung injury. Am J Physiol Lung Cell Mol Physiol 2008; 295: L379-L399.

56 Laffey JG, Kavanagh BP. Insight into acute respiratory distress syndrome: from models to patients. Am J Respir Crit Care Med 2017; 196: 18-28.

57 Wong HR, Caldwell JT, Cvijanovich NZ, et al. Prospective clinical testing and experimental validation of the Pediatric Sepsis Biomarker Risk model. Sci Transl Med 2019; 11: eaax9000.

58 Famous KR, Delucchi K, Ware LB, et al. Acute respiratory distress syndrome subphenotypes respond differently to randomized fluid management strategy. Am J Respir Crit Care Med 2017; 195: 331-338.

59 Sinha P, Delucchi KL, Thompson BT, et al. Latent class analysis of ARDS subphenotypes: a secondary analysis of the statins for acutely injured lungs from sepsis (SAILS) study. Intensive Care Med 2018; 44: 1859-1869.

60 Calfee CS, Delucchi KL, Sinha P, et al. Acute respiratory distress syndrome subphenotypes and differential response to simvastatin: secondary analysis of a randomised controlled trial. Lancet Respir Med 2018; 6: 691-698

61 Delucchi K, Famous KR, Ware LB, et al. Stability of ARDS subphenotypes over time in two randomised controlled trials. Thorax 2018; 73: 439-445.

62 Bos LDJ, Schouten LR, van Vught LA, et al. Identification and validation of distinct biological phenotypes in patients with acute respiratory distress syndrome by cluster analysis. Thorax 2017; 72: 876-883. 
63 Bos LD, Scicluna BP, Ong DY, et al. Understanding heterogeneity in biological phenotypes of ARDS by leukocyte expression profiles. Am J Respir Crit Care Med 2019; 200: 42-50.

64 Sinha P, Delucchi KL, Mcauley DF, et al. Development and validation of parsimonious algorithms to classify acute respiratory distress syndrome phenotypes: a secondary analysis of randomised controlled trials. Lancet Respir 2020; 2600: $1-11$.

65 Sinha P, Churpek MM, Calfee CS. Machine learning classifier models can identify ARDS phenotypes using readily available clinical data. Am J Respir Crit Care Med 2020; 202: 996-1004.

66 Prescott AHC, Calfee CS, Taylor B, et al. Towards smarter lumping and smarter splitting: rethinking strategies for sepsis and ARDS clinical trial design. Am J Respir Crit Care Med 2016; 112018: 1-28.

67 Samanta RJ, Summers C. Translational research in intensive care unit: novel approaches for drug development and personalized medicine. Semin Respir Crit Care Med 2019; 40: 687-698.

68 Mrozek S, Jabaudon M, Jaber S, et al. Elevated plasma levels of sRAGE are associated with nonfocal CT-based lung imaging in patients with ARDS: a prospective multicenter study. Chest 2016; 150: 998-1007.

69 Calfee CS, Janz DR, Bernard GR, et al. Distinct molecular phenotypes of direct vs indirect ARDS in single-center and multicenter studies. Chest 2015; 147: 1539-1548.

70 Fremont RD, Koyama T, Calfee CS, et al. Acute lung injury in patients with traumatic injuries: utility of a panel of biomarkers for diagnosis and pathogenesis. J Trauma 2010; 68: 1121-1127.

71 Cresci S, Pereira NL, Ahmad F, et al. Heart failure in the era of precision medicine: a scientific statement from the American Heart Association. Circ Genomic Precis Med 2019; 12: 458-485.

72 Agusti A, Bel E, Thomas $\mathrm{M}$, et al. Treatable traits: toward precision medicine of chronic airway diseases. Eur Respir J 2016; 47: 410-419.

73 Kitsios GD, Yang L, Manatakis D V, et al. Host-response subphenotypes offer prognostic enrichment in patients with or at risk for acute respiratory distress syndrome. Crit Care Med 2019; 27: 1724-1734.

74 Bos LDJ, Azoulay E, Martin-Loeches I. Future of the ICU: finding treatable needles in the data haystack. Intensive Care Med 2019; 45: 240-242.

75 Wells AU, Hirani N. Interstitial lung disease guideline: the British Thoracic Society in collaboration with the Thoracic Society of Australia and New Zealand and the Irish Thoracic Society. Thorax 2008; 63: v1-58.

76 Faverio P, De Giacomi F, Sardella L, et al. Management of acute respiratory failure in interstitial lung diseases: overview and clinical insights. BMC Pulm Med 2018; 18: 1-13.

77 Taniguchi H, Kondoh Y. Acute and subacute idiopathic interstitial pneumonias. Respirology 2016; 21: 810-820.

78 Phua J, Dean NC, Guo Q, et al. Severe community-acquired pneumonia: timely management measures in the first 24 hours. Crit Care 2016; 20: 237.

79 National Heart Lung and Blood Institute Acute Respiratory Distress Syndrome (ARDS) Clinical Trials Network, Wiedemann HP, Wheeler AP, et al. Comparison of two fluid-management strategies in acute lung injury. $N$ Engl $J$ Med 2006; 354: 2564-2575.

80 Determann RM, Royakkers A, Wolthuis EK, et al. Ventilation with lower tidal volumes as compared with conventional tidal volumes for patients without acute lung injury: a preventive randomized controlled trial. Crit Care 2010; 14: R1.

81 Zampieri FG, Póvoa P, Salluh JI, et al. Lower respiratory tract infection and short-term outcome in patients with acute respiratory distress syndrome. J Intensive Care Med 2020; 35: 588-594.

82 Sinha P, Calfee CS, Beitler JR, et al. Physiological analysis and clinical performance of the ventilatory ratio in acute respiratory distress syndrome. Am J Respir Crit Care Med 2019; 199: 333-341.

83 Amato MBP, Meade MO, Slutsky AS, et al. Driving pressure and survival in the acute respiratory distress syndrome. N Engl J Med 2015; 372: 747-755.

84 Constantin JM, Grasso S, Chanques G, et al. Lung morphology predicts response to recruitment maneuver in patients with acute respiratory distress syndrome. Crit Care Med 2010; 38: 1108-1117.

85 Jabaudon M, Blondonnet R, Lutz J, et al. Net alveolar fluid clearance is associated with lung morphology phenotypes in acute respiratory distress syndrome. Anaesth Crit Care Pain Med 2016; 35: 81-86.

86 Famous KR, Delucchi K, Ware LB, et al. ARDS subphenotypes respond differently to randomized fluid management strategy. Am J Respir Crit Care Med 2017; 194: 331-338.

87 Jones TK, Feng R, Kerchberger VE, et al. Plasma sRAGE acts as genetically regulated causal intermediate in sepsis-associated ARDS. Am J Respir Crit Care Med 2020; 201: 47-56.

88 Agrawal A, Matthay MA, Kangelaris KN, et al. Plasma angiopoietin-2 predicts the onset of acute lung injury in critically ill patients. Am J Respir Crit Care Med 2013; 187: 736-742.

89 Chang JC. Acute respiratory distress syndrome as an organ phenotype of vascular microthrombotic disease: based on hemostatic theory and endothelial molecular pathogenesis. Clin Appl Thromb 2019; 25: 1076029619887437.

90 Patel BV, Arachchillage DJ, Ridge CA, et al. Pulmonary angiopathy in severe COVID-19: physiologic, imaging and hematologic observations. Am J Respir Crit Care Med 2020; 202: 690-699.

91 Morrell ED, Radella F, Manicone AM, et al. Peripheral and alveolar cell transcriptional programs are distinct in acute respiratory distress syndrome. Am J Respir Crit Care Med 2018; 197: 528-532.

92 Bos L, Sinha P, Dickson R. The perils of premature phenotyping in COVID: a call for caution. Eur Respir J 2020; 56: 2001768 . 